\title{
Observation of "Ghost" Islands and Surfactant Effect of Surface Gallium Atoms during GaN Growth by Molecular Beam Epitaxy
}

\author{
L. X. Zheng, ${ }^{1,2}$ M. H. Xie, ${ }^{1}$ S. M. Seutter, ${ }^{1}$ S. H. Cheung, ${ }^{1}$ and S. Y. Tong ${ }^{1}$ \\ ${ }^{1}$ Department of Physics, University of Hong Kong, Pokfulam Road, Hong Kong \\ ${ }^{2}$ State Key Laboratory on Integrated Optoelectronics, Institute of Semiconductors, Chinese Academy of Sciences, Beijing, China
}

(Received 26 April 2000)

\begin{abstract}
We observe "ghost" islands formed on terraces during homoepitaxial nucleation of GaN. We attribute the ghost islands to intermediate nucleation states, which can be driven into "normal" islands by scanning tunneling microscopy. The formation of ghost islands is related to excess $\mathrm{Ga}$ atoms on the surface. The excess Ga also affect island number density: by increasing Ga coverage, the island density first decreases, reaching a minimum at about 1 monolayer (ML) Ga and then increases rapidly for coverages above 1 ML. This nonmonotonic behavior points to a surfactant effect of the Ga atoms.
\end{abstract}

PACS numbers: 68.55.-a, 61.16.Ch, 68.35.Bs, 81.10.-h

The growth of GaN films has attracted much attention because of applications that this material has in fabricating intense blue/green light emitting diodes, lasers, and high temperature/power electronic devices [1-3]. However, until recently, little is known of the microscopic details of the growth process. Recent experiments have shown that for the Ga-terminated face, i.e., GaN (0001), the " $1 \times 1$ " surface is terminated by up to 2 monolayers (ML) of Ga adatoms beyond the last bilayer. The top-layer excess $\mathrm{Ga}$ atoms are thought to exist in a completely disordered, fluid phase [4]. It has also been established that film growth under excess-Ga conditions generally results in better quality films with a smoother surface morphology. Thus, it seems that the excess $\mathrm{Ga}$ atoms on the surface of a film play an important, if not fully understood, role in affecting the microscopic processes governing growth.

The purpose of this Letter is to report a novel observation of an intermediate nucleation state occurring at the surface of the film. Using scanning tunneling microscopy (STM) to study GaN (0001) films grown by molecular beam epitaxy (MBE), we observe "ghost" islands on surfaces grown under excess $\mathrm{Ga}$ conditions. The ghost islands can be irreversibly driven into normal islands by simply continuous imaging or altering scanning voltage of the STM (at no time does the tip touch the sample). Thus, we associate the ghost islands to a metastable, intermediate nucleation state of the surface. We further establish that the ghost islands appear only when there are excess $\mathrm{Ga}$ atoms on the surface. An increase in $\mathrm{N}$ deposition changes the ghost islands into normal islands. Details of the experimental conditions and these observations are given below.

A second set of observations is that the island number density during growth depends sensitively on the amount of excess $\mathrm{Ga}$ atoms on the surface. The island density first decreases with increasing excess Ga coverage, up to $1 \mathrm{ML}$. This indicates that in the less than $1 \mathrm{ML}$ regime, excess $\mathrm{Ga}$ atoms enhance diffusion of the arriving $\mathrm{N}$ atoms. How- ever, at excess Ga coverages greater than $1 \mathrm{ML}$, the island density rapidly increases. This result suggests a surfactantmediated growth process. Surfactant-mediated growth is a recently discovered phenomenon where the growth mode can be changed from three-dimensional (3D) islanding to 2D layer-by-layer growth $[5,6]$. It is achieved by depositing on a substrate $1 \mathrm{ML}$ or more of a third material, different from the substrate and the epilayer. During growth, this third material or "surfactant layer" will decrease the surface energy and thus float on top through an exchange action between atoms of the surfactant layer and the deposit. The presence of a surfactant layer on the surface can change the growth kinetics from the traditional diffusion-limited process $[7,8]$ to an exchange reaction-limited process [9-11]. Consequently, it leads to very different growth behaviors, one of which is an increase in the island number density. However, unlike the conventional surfactant-mediated growth, the new observation here is that the surfactant is itself a constituent component of the GaN film. This novel process has the advantage of improving a film's quality during growth but avoiding undesirable incorporation of foreign (surfactant) atoms in the film.

The experiments are conducted in a multifunctional ultrahigh vacuum system containing, among other interconnected chambers, an MBE reactor and an STM system. The MBE chamber consisted of an effusive cell for Ga source and a radiofrequency plasma generator (Oxford Applied Research, CARS-25) for N. Prior to GaN deposition at low temperatures, a thick GaN buffer layer is first grown on a $\mathrm{SiC}(0001)$ substrate at $650{ }^{\circ} \mathrm{C}$ under the excess-Ga condition. The buffer layer grown under such conditions has atomically flat surfaces with relatively large terraces. The film has the Ga polarity as judged by surface reconstruction observations [12,13]. The buffer layer growth is interrupted by closing the source shutters, and the substrate temperature is lowered to $\sim 400-500{ }^{\circ} \mathrm{C}$, conditions which promote $2 \mathrm{D}$ island nucleation [12] due to reduced 
diffusion length of adatoms on the surface. GaN growth is reinitiated at the low temperatures for a specified period before the sample is thermally quenched and transferred to the STM chamber for analysis. Room temperature STM experiments are carried out at a tunneling current of $0.1 \mathrm{nA}$ and a sample bias of $-3.0 \mathrm{~V}$, unless stated otherwise.

Figure 1(a) shows a typical STM image of the surface following growth under excess $\mathrm{Ga}$ conditions and at low temperatures. It is seen that triangularly shaped islands populate the terraces of the buffer layer. The faster growth speeds of double-bonded bilayer edges versus single-bonded bilayer edges result in the triangularly shaped islands, as explained in an earlier paper [14]. Measurements of height differences by STM confirm that the terraces have single bilayer height differences and the triangular islands have single bilayer heights on the terraces themselves. What is surprising in Fig. 1(a) is the presence of ghost islands having low STM contrasts, especially in the interior of the islands. The ghost islands have the triangular shape, with the inside appearing "hollow" and surrounded by bright frames. Moreover, the ghost

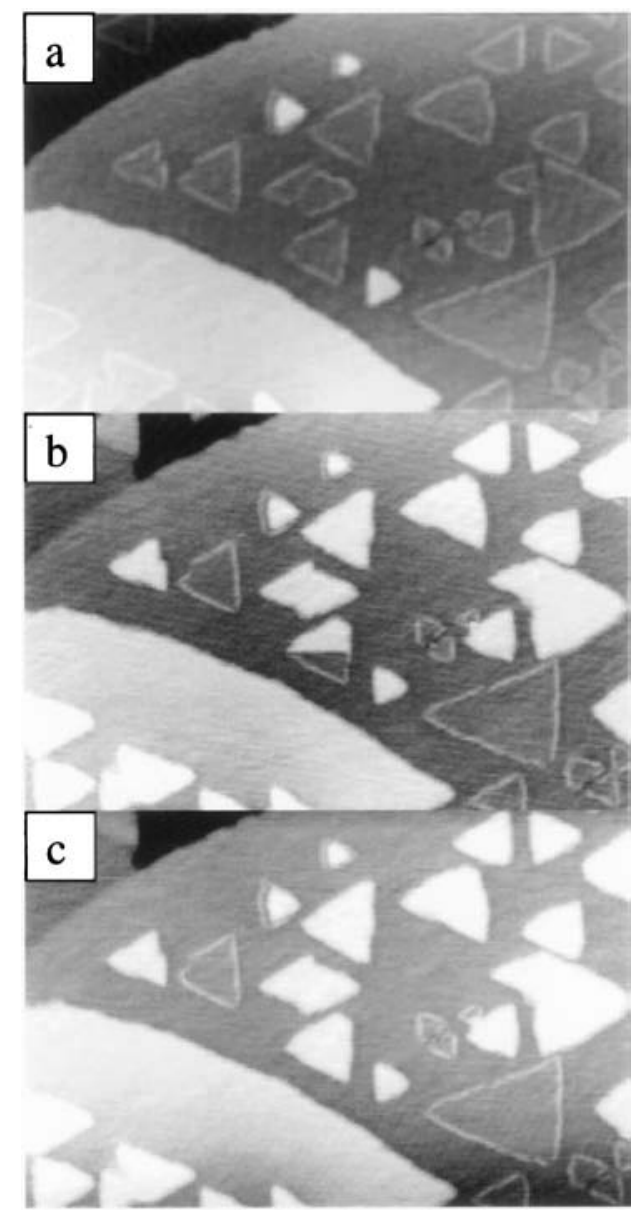

FIG. 1. STM images of GaN surface (a) immediately after the growth; (b) after reversing the gap voltage to $+2.5 \mathrm{~V}$; and (c) after restoring the original scanning condition as used in (a). The image sizes are $135 \mathrm{~nm} \times 75 \mathrm{~nm}$ for all three images. islands can be converted into normal, bilayer-height islands following continuous STM imaging with or without altering the imaging conditions. An example showing the converted islands by simply changing the sample-tip voltage is presented in Fig. 1(b). There is a clear oneto-one correspondence between the ghost and "normal" islands. The converted normal islands have the same island height (1 bilayer) and the distinct triangular shape. It is further found that island conversion is irreversible; i.e., once a ghost island has been converted to a normal island, it will remain normal and cannot be changed back to a ghost through STM imaging. An STM image of the same surface obtained immediately after Fig. 1(b) but using the same imaging voltage as in Fig. 1(a) is shown in Fig. 1(c). In Figs. 1(b) and 1(c), some ghost islands have not yet been converted. However, with additional STM scanning, all ghost islands will be eventually converted into normal islands.

Since the normal and ghost islands are both present and randomly scattered in an STM image, we cannot attribute the ghosts to an STM artifact, although the scanning can induce a conversion as demonstrated in Figs. 1(a)-1(c). Further experiments show that lowering the growth temperature and increasing Ga coverage will increase the population of ghost islands. Another interesting finding is that when growth is under the stoichiometric or excess $\mathrm{N}$ condition, only normal islands are observed by STM. Thus, we believe that the ghost islands are real surface features related to the presence of excess $\mathrm{Ga}$ atoms on the surface. Because of the one-to-one correspondence between ghost and normal islands and the irreversible conversion demonstrated above, we suggest that the ghost islands probably correspond to an intermediate nucleation state involving $\mathrm{Ga}$ atoms. This intermediate nucleation state is converted to a normal state by STM scanning. We now report experimental observations which suggest that the ghost islands play an important role in the growth kinetics of GaN films.

To show this, we have conducted a series of experiments in which $\mathrm{Ga}$ and $\mathrm{N}$ are deposited alternately rather than at the same time. The same procedure, known as "atomic layer epitaxy," has been used to study the growth of II-VI compounds [15]. Figure 2 shows STM images of surfaces following different $\mathrm{N}$ exposures but having the same excess $\mathrm{Ga}$ coverage. The starting surfaces are prepared by growing thick $\mathrm{GaN}$ buffer films under the identical excess Ga condition and for the same time interval. It is interesting to observe that following a $0.14 \mathrm{ML}$ deposition of $\mathrm{N}$, the surface shows sizable islands; some are normal while most others are mixtures of normal islands formed inside "ghostlike" triangles [Fig. 2(a)]. By increasing the $\mathrm{N}$ exposure to $0.3 \mathrm{ML}$, almost all the islands are normal, as shown in Fig. 2(b). Further increase of the $\mathrm{N}$ exposure produces little change in the characteristics of the islands compared to that of Fig. 2(b), including the island areal density. Figures 2(c) and 2(d) are close-up STM images 


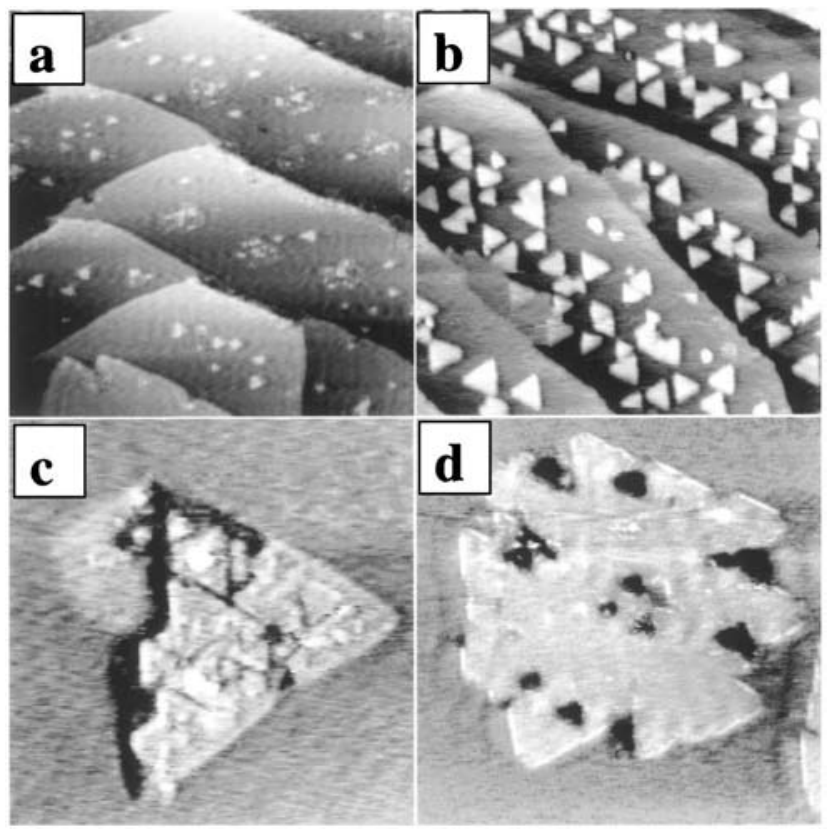

FIG. 2. STM images after exposing the Ga-terminated GaN surfaces to $\mathrm{N}$ for (a) $0.14 \mathrm{ML}$ and (b) $0.30 \mathrm{ML}$ at $450{ }^{\circ} \mathrm{C}$. The image sizes are $200 \mathrm{~nm} \times 200 \mathrm{~nm}$. (c),(d) Close-up images $(40 \mathrm{~nm} \times 40 \mathrm{~nm})$ of the surface similar to (a) showing morphological features of partially converted islands.

of a surface similar to Fig. 2(a), which show detailed morphological features of the partially converted islands. In Fig. 2(c), converted normal islands are seen residing in a large triangular area partially bordered by voids. The islands appear fragmented. If the normal islands in the triangular areas are formed by converting a triangular ghost seen in Fig. 1(a), then Fig. 2(c) suggests a conversion process which occurs in parts rather than as a whole for the ghost island. These observations indicate possibly different mechanisms between the conversion of ghost to normal islands by STM scanning versus N deposition. Figure 2(d) shows another morphological feature of the $\mathrm{N}$-deposition converted islands. The fractal-like normal island becomes dominant when the rate of deposition is lowered, in agreement with recent kinetic Monte Carlo (KMC) simulations when an exchange process between surfactant and deposit is considered [11].

Figure 3 shows a series of STM images of surfaces following various $\mathrm{Ga}$ depositions but at the same $\mathrm{N}$ exposure. To achieve this, we first prepare GaN buffer films (at $650{ }^{\circ} \mathrm{C}$ ) under the stoichiometric condition [12], followed by deposition of different amounts of Ga from the Knudsen cell at low sample temperatures $\left(400-500{ }^{\circ} \mathrm{C}\right)$. The surfaces are then exposed to a fixed amount $(0.25 \mathrm{ML})$ of $\mathrm{N}$ before thermal quenching for STM analysis. Figures 3(a)-3(d) show the resulting surfaces for Ga coverages of $0.4,1.0,1.5$, and $3 \mathrm{ML}$, respectively. Several distinct features are revealed: (1) For a Ga coverage of less than 1 ML [Fig. 3(a)], the majority of islands appear normal, which contrasts the situation where the Ga coverage is

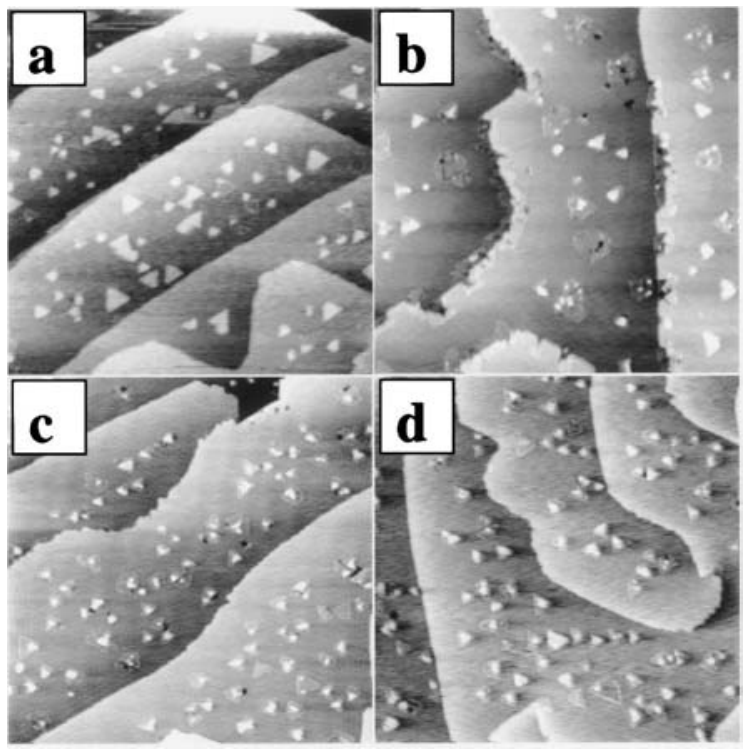

FIG. 3. STM images of GaN surfaces after depositing (a) $0.4 \mathrm{ML}$, (b) $1 \mathrm{ML}$, (c) $1.5 \mathrm{ML}$, and (d) $3 \mathrm{ML}$ Ga and followed by $0.25 \mathrm{ML}$ deposition of $\mathrm{N}$ at $450^{\circ} \mathrm{C}$. The image sizes are $200 \mathrm{~nm} \times 200 \mathrm{~nm}$ for all images.

above $1 \mathrm{ML}$, with most islands being either ghosts or partially converted normal-ghost mixtures [Figs. 3(b)-3(d)]. (2) As the Ga coverage increases, the island density first decreases, reaching a minimum at about the 1 ML coverage. After that, the island density increases at a rapid rate and saturates when the coverage is about 2 ML. The island density versus excess Ga coverage is plotted in Fig. 4. Feature (1) is consistent with the observation made in Figs. 1 and 2; i.e., ghost and normal-ghost mixed islands form only where there is excess Ga on the surface. Feature (2) shows that the excess Ga atoms behave as a surfactant that has a profound effect on nucleation.

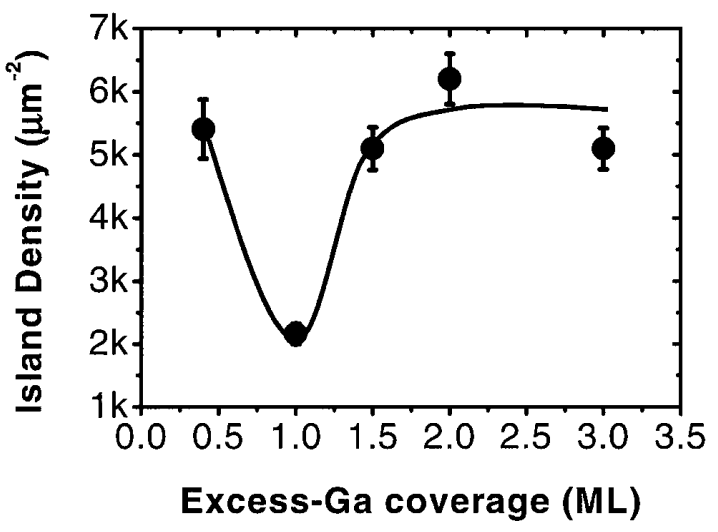

FIG. 4. Island density dependence on excess Ga coverage. The island densities (filled circles) are calculated according to $1 / l^{2}$, where $l$ is the island-island distance away from the region near the surface steps, therefore, excluding the effect of steps on nucleation. The error bars represent standard deviation of the mean, while the solid line is drawn to guide the eye. 
Recent theoretical and KMC simulation studies $[9,11]$ of surfactant-mediated growth have indicated modifications to classical nucleation theory $[7,8]$. The surfactant atoms can block surface reaction sites; therefore, nucleation invokes site exchange action between the deposited and surfactant atoms. The exchange action requires overcoming an energy barrier leading to grossly different island density distribution, as well as novel island shape transition from fractal to compact [10,11]. Our observations of triangularly shaped ghost islands and fractal-like normal or mixed islands are thus consistent with such predictions. It can be inferred that excess $\mathrm{Ga}$ atoms on a GaN surface act as surfactants for epitaxial growth. Indeed, it has been observed that up to $2 \mathrm{ML}$ of Ga exist and wet the surface of $\mathrm{SiC}$ substrates [16] and $\mathrm{GaN}$ epifilms [4]. In order for $\mathrm{GaN}$ epitaxy to proceed, the incoming $\mathrm{N}$ atoms must exchange sites with the surfactant $\mathrm{Ga}$ atoms. Such an exchange can be hindered at low temperatures by an exchange barrier. According to this scenario, when the surfactant Ga layer is less than a certain coverage, the deposited N atoms can easily "see" the GaN surface and growth proceeds without invoking site-exchange action. Classical nucleation theory applies and the presence of excess surface $\mathrm{Ga}$ atoms enhances surface diffusion, leading to fewer islands being nucleated in this regime. This explains the initial decrease of island density versus $\mathrm{Ga}$ coverage up to this critical value. When the Ga coverage is above the critical value, the GaN surface becomes fully "hidden" and the nucleation is limited by a "surfactantdeposit" exchange rate. This manifests in an increase in the island density, as shown in Fig. 4. The increase is explained by the fact that already-nucleated islands may not effectively trap the surface diffusing adatoms, because of the exchange barrier. Adatoms then have more chances to form new islands near preexisting islands. As suggested by Kandel [9], adatoms do not see the step edges of an existing island for incorporation; therefore, they nucleate new islands on the terraces, resulting in more islands. From Fig. 4, the critical coverage is about $1 \mathrm{ML}$, although the exact value is not accurately determined due to the lack of data points between 0.5-1 ML.

To summarize, nucleation of GaN during MBE growth shows a novel behavior under the excess Ga growth condition. STM images show ghost islands and these are attributed to an intermediate nucleation state. The overall island density is found to depend critically on the surface $\mathrm{Ga}$ coverage. The results point to a surfactant effect of the excess Ga atoms. Depending on the coverage, the kinetics are different: at less than $1 \mathrm{ML}$ coverage, classical nucleation theory applies; however, above 1 ML, exchange and incorporation rates of adatoms limit growth. The two regimes result in a nonmonotonic behavior of the island density versus $\mathrm{Ga}$ coverage. From a practical viewpoint, the surfactant effect of $\mathrm{Ga}$ atoms may be utilized to better control growth, thereby improving the films' quality. However, unlike traditional surfactant-mediated growth, where a third material is added to the growing surface, $\mathrm{Ga}$ is a constituent component of $\mathrm{GaN}$, thus having the added advantage of avoiding contamination by surfactant atoms in films. Finally, we point out that at high temperatures of MBE, the growth mode becomes step-flow and the surfactant effect observed at low temperatures becomes less evident.

The authors thank Ho Win kin for technical support, S. J. Xu, H. S. Wu, and X. Q. Dai for fruitful discussions. This study is supported in part by HK RGC Grants No. HKU7118/98P, No. 7142/99P, and No. 260/95P.

[1] S. Strite and H. Morkoc, J. Vac. Sci. Technol. B 10, 1237 (1992).

[2] S. Nakamura, T. Mukai, and M. Senish, Appl. Phys. Lett. 64, 1687 (1994).

[3] I. Akasaki, H. Amono, S. Sota, H. Sakai, T. Tanaka, and M. Koike, Jpn. J. Appl. Phys. 34, L1517 (1995).

[4] A. R. Smith, R. M. Feenstra, D. W. Greve, M.S. Shin, M. Skowronski, J. Neugebauer, and J. E. Northrup, J. Vac. Sci. Technol. B 16, 2242 (1998).

[5] M. Copel, M.C. Reuter, E. Kaxiras, and R. M. Tromp, Phys. Rev. Lett. 63, 632 (1989).

[6] M. H. von Hoegen, F. K. Legoues, M. Copel, M. C. Reuter, and R. M. Tromp, Phys. Rev. Lett. 67, 1130 (1991).

[7] J. A. Venables, G. D. T. Spiller, and M. Hanbücken, Rep. Prog. Phys. 47, 399 (1984).

[8] M.C. Bartelt and J. W. Evans, Phys. Rev. B 46, 12675 (1992).

[9] D. Kandel, Phys. Rev. Lett. 78, 499 (1997).

[10] T.-C. Chang, I.-S. Hwang, and T. T. Tsong, Phys. Rev. Lett. 83, 1191 (1999).

[11] B.-G. Liu, J. Wu, E. G. Wang, and Z. Zhang, Phys. Rev. Lett. 83, 1195 (1999).

[12] S. M. Seutter, M.H. Xie, W. K. Zhu, L.X. Zheng, Huasheng Wu, and S. Y. Tong, Surf. Sci. 445, L71 (2000).

[13] A. R. Smith, R. M. Feenstra, D. W. Greve, M.-S. Shin, M. Skowronski, J. Neugebauer, and J. E. Northrup, Appl. Phys. Lett. 72, 2114 (1998).

[14] M.H. Xie, S.M. Seutter, W. K. Zhu, L.X. Zheng, Huasheng Wu, and S. Y. Tong, Phys. Rev. Lett. 82, 2749 (1999).

[15] M. Ahonen, M. Pessa, and T. Suntola, Thin Solid Films 65, 301 (1980).

[16] L. X. Zheng, M. H. Xie, and S. Y. Tong, Phys. Rev. B 61, 4890 (2000). 